\title{
Poly(amido amine) is an ideal carrier of miR-7 for enhancing gene silencing effects on the EGFR pathway in U251 glioma cells
}

\author{
XIAOZHI LIU ${ }^{1,3}$, GANG LI $^{1,3}$, ZHIGUO SU ${ }^{1,3}$, ZHONGMIN JIANG ${ }^{2}$, \\ LEI CHEN $^{1,3}$, JUNFEI WANG ${ }^{1,3}$, SHIZHU YU ${ }^{4,5}$ and ZHENLIN LIU ${ }^{1,3}$
}

\begin{abstract}
Departments of ${ }^{1}$ Neurosurgery, and ${ }^{2}$ Pathology, The Fifth Central Hospital of Tianjin, Tianjin; ${ }^{3}$ Tianjin Binhai Neurological Institute, Tianjin; ${ }^{4}$ Department of Neuropathology Institute, Department of Tianjin Neurological, Tianjin Medical University General Hospital, Tianjin; ${ }^{5}$ Key Laboratory of Post-trauma Neuro-repair and Regeneration in Central Nervous System, Ministry of Education; Tianjin Key Laboratory of Injuries, Variations and Regeneration of Nervous System, Tianjin, P.R. China
\end{abstract}

Received July 25, 2012; Accepted November 27, 2012

DOI: $10.3892 / o r .2013 .2283$

\begin{abstract}
RNAs are regarded as promising drugs for glioma gene therapy. However, conventional administration routes, such as oral administration and intravenous infusion, present low efficiency due to the blood-brain barrier and intercellular retention, thereby limiting their application. Recent studies showed poly(amido amine) (PAMAM) was a candidate carrier due to its high solubilization, delayed release and low toxicity. In the present study, U251 human brain glioma cells were transfected with the miR-7 gene using PAMAM as the vector to determine the transfection efficiency and therapeutic effects in vivo and in vitro. We found that PAMAM exhibited higher transfection efficiency and longer duration of action compared with liposome delivery, and miR-7 efficiently silenced some genes involved in the epidermal growth factor receptor (EGFR) pathway and achieved favorable effects in treating glioma in vivo and in vitro. These investigations provide a basis for developing high-efficiency micromolecular drug delivery.
\end{abstract}

\section{Introduction}

Glioma is a type of primary tumor that starts in the brain or spine and accounts for $2 \%$ of tumors in adults. The 5 -year case fatality rate is the highest of any cancer except for pancreatic and lung carcinoma (1-3). Treatment is often a combined approach, using surgery, radiation therapy and chemotherapy, however, these treatments cannot significantly improve the

Correspondence to: Professor Zhenlin Liu, Department of Neurosurgery, The Fifth Central Hospital of Tianjin, 41 Zhejiang Road, Tanggu District, Tianjin 300450, P.R. China

E-mail: wjzhenlin817@sina.com

Key words: microRNA-7, glioma, poly(amido amine), folic acid, epidermal growth factor receptor survival rate beyond 5 years and, over the past 30 years, the median survival duration of glioblastoma has been $<1$ year $(4,5)$.

Glioma is a disease involving abnormal genes. Epidermal growth factor receptor (EGFR) mutation, amplification and overexpression are early molecular events for glioma progression. EGFR facilitates the cell cycle of tumor cells, inhibits cell apoptosis, maintains telomerase activity and promotes tumor angiogenesis and tumor invasion through downstream pathways (6-9).

microRNAs are a class of endogenous small non-coding RNAs that regulate gene expression by targeting mRNAs for translational repression, degradation, or both, through target sites in the $3^{\prime}$ untranslated regions ( $3^{\prime}$ UTR) and modulate cell differentiation, proliferation and apoptosis (10-13). Kefas et al first reported complementarity in at least three regions in the $3^{\prime}$ UTR between miR-7 and EGFR (14). Previous studies by our group indicated that miR-7 significantly inhibited glioma cell line proliferation and partially silenced EGFR post-transcriptional translation and reduced EGFR activity and function (15). However, animal experiments showed that neither liposomeor adenovirus-transfected miR-7 can effectively decrease the survival rate of rats with glioma, although survival duration was slightly prolonged. Subsequent experiments revealed that due to the blood-brain barrier and intercellular retention, target genes in the tumor bed were poorly targeted, significantly limiting the function of miR-7. Thus, a gene delivery material that can pass through the blood-brain barrier and is independent of intercellular communication is needed.

Poly(amido amine) (PAMAM) has a large volume and a large number of surface functional groups. The high degree of branching and the specific monodispersity property allow the terminal functional groups to include anion, cation and hydrophobic groups, significantly improving biocompatibility, bioavailability and targeting. In addition, due to its high solubilization, delayed release and low toxicity, PAMAM has been used as a drug delivery system (16-18). Folic acid (FA) is rarely expressed in epithelial cells of newly generated normal tissues, but is highly expressed in a variety of tumor cells and 
positively correlates with tumor malignancy. Therefore, FA has become a target for tumor cell tracing $(19,20)$. The present study utilized PAMAM as a vector and FA/FA receptor as a target axis and found that miR-7 efficiently silenced gliomaspecific genes and achieved favorable effects in treating glioma in vivo and in vitro.

\section{Materials and methods}

Cell culture conditions. U251 human glioma cells were kindly provided by the Tianjin Neuro-Oncology Institute, China. All cells were maintained in Dulbecco's modified Eagle's medium (DMEM; Invitrogen, Carlsbad, CA, USA) supplemented with $10 \%$ fetal bovine serum (FBS; Invitrogen), $2 \mathrm{mM}$ glutamine (Sigma, St. Louis, MO, USA), 100 units of penicillin/ml (Sigma) and $100 \mu \mathrm{g}$ of streptomycin $/ \mathrm{ml}$ (Sigma), at $37^{\circ} \mathrm{C}$ with $5 \% \mathrm{CO}_{2}$.

Animals. A total of 174 male athymic mice, weighing $80 \mathrm{~g}$, were purchased from the Animal Center of the Academy of Military Medical Sciences, Beijing (License no. 0195), China, and housed in the Animal Experimental Center, Tianjin Medical University, with a humidity of $50 \pm 5 \%$ at $20-25^{\circ} \mathrm{C}$.

FA/PAMAM complex preparation and transmission electron microscopy observations. FA (Sigma) was linked to PAMAM-D (Sigma) through two continuous steps. Briefly, $0.03278 \mathrm{~g}$ FA was reacted with $0.19979 \mathrm{~g}$ EDC in $20 \mathrm{ml}$ dimethyl sulphoxide and the FA activated solution was gradually dropped into a PAMAM-methanol and dimethyl sulphoxide solution. The complex produced was observed using transmission electron microscopy.

Cell transfection. The miR-7 sequence was 5'-UUGUUUUA GUGAUCAAGAAGGU-3' and the reference antisense strand was 5'-GTGAATGCGATGCCCTCCGAGG-3'. The 5' end was labeled with fluorescein isothiocyanate. There were three groups in the experiments: normal control, FA/PAMAM/ nonsense-treated and FA/PAMAM/miR-7 treated. When U251 cells reached $60-70 \%$ confluency, serum concentration was decreased to 5\% and PAMAM and miR-7 or nonsense control were added at a ratio of $16: 1$ and cultured at $37^{\circ} \mathrm{C}$ in $5 \%$ $\mathrm{CO}_{2}$ for $4 \mathrm{~h}$. The culture medium was replaced with complete culture medium supplemented with $10 \%$ FBS and cells were incubated for an additional $48 \mathrm{~h}$. Cell transfection was observed using a fluorescence microscope.

Reverse transcription (RT)-real-time PCR. RT reactions and real-time PCR were conducted using the mirVana ${ }^{\mathrm{TM}}$ qRT-PCR miRNA detection kit (Ambion, Austin, TX, USA). Amplification was performed using an MJ-real-time PCR (Bio-Rad Laboratories, Hercules, CA, USA) with a protocol of 40 cycles at $95^{\circ} \mathrm{C}$ for $3 \mathrm{~min}, 95^{\circ} \mathrm{C}$ for $15 \mathrm{sec}$ and $60^{\circ} \mathrm{C}$ for $30 \mathrm{sec}$. miR-7 upstream primer: 5'-AAAAAGAACACGTGGAAGGAT AG-3'; downstream primer: 5'-CCGCCTAACGTACCGCG AATTT-3'. A human 18 rRNA TaqMan probe was used as an internal reference, with upstream primer: 5'-AACTTTCGAT GGTAGTCGCCG-3' and downstream primer: 5'-CCTTGGA TGTGGTAGCCGTTT-3'. Both RT and PCR primers were purchased from Ambion. 5S RNA was used for normalization.
Relative quantification was conducted using amplification efficiencies derived from cDNA standard curves. Data were analyzed using Opticon Monitor Analysis software V2.02 (MJ Research Inc., Waltham, MA, USA) and are shown as foldchange $\left(2^{-\Delta \Delta C t}\right)$.

Western blot analysis. Parental and transfected cells were washed three times with pre-chilled phosphate-buffered saline (PBS). The cells were then solubilized in $1 \%$ Nonidet P-40 lysis buffer (20 mM Tris, $\mathrm{pH} 8.0,137 \mathrm{mM} \mathrm{NaCl}, 1 \%$ Nonidet P-40, $10 \%$ glycerol, $1 \mathrm{mM} \mathrm{CaCl}_{2}, 1 \mathrm{mM} \mathrm{MgCl}_{2}, 1 \mathrm{mM}$ phenylmethylsulfonyl fluoride, $1 \mathrm{mM}$ sodium fluoride, $1 \mathrm{mM}$ sodium orthovanadate and a protease inhibitor mixture). Lysates (40 $\mu \mathrm{g}$ ) were separated by $8 \%$ SDS-acrylamide electrophoresis. Separated proteins were transferred to polyvinylidene fluoride membranes (Millipore, Bedford, MA, USA) and incubated with primary antibodies against EGFR (1:500 dilution; Santa Cruz Biotechnology, Santa Cruz, CA, USA), Akt-2 (1:1,000 dilution; for Ser473, Santa Cruz Biotechnology), and PI3K (Zhongshan Bio Corp., Beijing, China), followed by incubation with horseradish peroxidase-conjugated secondary antibody (Zymed Laboratories Inc., San Francisco, CA, USA). Specific proteins were detected using a SuperSignal protein detection kit (Pierce, Rockford, IL, USA). The membrane was stripped and reprobed with a primary antibody against $\beta$-actin (Santa Cruz Biotechnology). Optical density was measured and analyzed using Quantity One software.

Cell growth curves. Cells were seeded into 24-well plates at $1 \times 10^{4}$ cells/well. The number of cells was quantified following culture for 1,2,3,4,5 and 6 days to produce cell growth curves.

Cell cycle detection. Single cell suspensions were obtained following trypsinization and cells were then washed with PBS three times, fixed with absolute alcohol and incubated with RNaseA. Cells were stained with propidium iodide for $30 \mathrm{~min}$ and then analyzed by flow cytometry. Samples were collected and analyzed using CellQuest software, and a proliferation index was calculated according to $\left[\mathrm{PIx}=\left(\mathrm{S}+\mathrm{G}_{2} \mathrm{M}\right) /\right.$ $\left.\left(\mathrm{G}_{0} \mathrm{G}_{1}+\mathrm{S}+\mathrm{G}_{2} \mathrm{M}\right)\right]$.

Apoptosis detection using the TUNEL method. Cells were mounted in PBS, immersed in permeabilization solution for 5 min, incubated with $25 \mu 1$ TUNEL-labeling reaction mixture in a humidified box at $37^{\circ} \mathrm{C}$ for $60 \mathrm{~min}$ and then washed with PBS. Nuclei were counterstained with Hoechst 33258 at a dilution of 1:1,000. Cell apoptosis rate (number of apoptotic cells/total number of cells x 100\%) was quantified using a fluorescence microscope.

Tumor cell migration detection. Matrigel $(60 \mu \mathrm{l})$ was placed on the polycarbonate membrane of the upper chamber of a Transwell at $37^{\circ} \mathrm{C}$ for $30 \mathrm{~min}$ and the gel was allowed to solidify. Cells were seeded into the upper Transwell chamber at $1 \times 10^{4}$ cells/well and $600 \mu 1$ DMEM supplemented with $10 \%$ FBS was added to the lower chamber. After $48 \mathrm{~h}$, cells that had not permeated the membrane in the upper chamber were removed using a wet cotton bud and the membrane was stained with hematoxylin. The number of cells permeating the membrane was quantified under an inverted microscope. 
Soft agar assay for clone formation. Six-well plates were precoated with agar $(0.7 \%)$ overnight. Stably transfected stem cell suspension was prepared, quantified and mixed with $0.35 \%$ low melting-point agarose prepared using DMEM containing $10 \% \mathrm{FBS}$, and then rapidly placed in the 6 -well plate and allowed to grow in a conventional incubator. Complete medium $(200 \mu \mathrm{l})$ was added to every well the following day and the medium was replaced every 7 days thereafter. After 3 weeks, the number of colonies with cells $>40$ was counted using an inverted microscope.

Establishment of a brain-glioma mouse model. In the liposome/miR-7 group, the serum concentration of the culture medium was decreased to $5 \%$, and $1 \mu \mathrm{g}$ plasmid was mixed with $100 \mu \mathrm{l} \mathrm{LipoVec}{ }^{\mathrm{TM}}$ for $20 \mathrm{~min}$ and then placed in the culture medium. After $4 \mathrm{~h}$, the medium was replaced with medium containing $20 \%$ serum. In the FA/PAMAM/miR-7 group, the serum concentration of the culture medium was decreased to 5\%. PAMAM and miR-7 (16:1) were added to $\mathrm{U} 251$ cells and incubated at $37^{\circ} \mathrm{C}$ in $5 \% \mathrm{CO}_{2}$. After $4 \mathrm{~h}$ of incubation, medium was replaced with medium supplemented with $10 \%$ FBS. In the U251 control group $(n=144)$, cells in the log phase of growth were harvested and suspended to prepare a single cell suspension. Mice were anesthetized by intraperitoneal injection with $10 \%$ chloral hydrate $(3.0 \mathrm{ml} / \mathrm{kg})$ and then fixed on a super-clean bench with stereotactic apparatus. Cell suspension $(5 \mu \mathrm{l})$ containing $1 \times 10^{6} \mathrm{U} 251$ cells was injected over $5 \mathrm{~min}$ into the right caudate nucleus. The needle was maintained in place for at least $3 \mathrm{~min}$ following injection and the incision was then sutured. All experimental procedures were carried out according to the regulations and internal biosafety and bioethics guidelines of Tianjin Medical University and the Tianjin Municipal Science and Technology Commission.

Cell transplantation and complex aggregation in the tumor. Drugs were administered 8 days after cell transplantation at three sites: caudal vein $(n=24)$; carotid artery $(n=24)$; and tumor site $(\mathrm{n}=24)$. Every mouse was administered $10 \mu 1$ FA/PAMAM solution containing $5 \mu \mathrm{g}$ nonsense sequence or miR-7 oligonucleotide. The mice were sacrificed 10 days after cell transplantation, i.e. $48 \mathrm{~h}$ after drug administration, and the brain was harvested to prepare frozen sections of tumor tissues. FA/PAMAM/miR-7 complex aggregation in the tumor was observed by fluorescence microscopy.

Comparison of glioma inhibition between two miR-7 delivery systems, both in vivo and in vitro gene transfection efficiency. Cells were obtained $48 \mathrm{~h}$ following gene transfection of liposome/miR-7, FA/PAMAM/miR-7 and U251 control groups. The percent of positive cells was determined by fluorescence microscopy and transfection efficiency was calculated.

miR-7 levels. Total-RNA was extracted from cells, transcribed with reverse transcriptase, followed by PCR using the same conditions as described above.

MRI. Seventy-two mice were examined. Each mouse was administered $10 \mu \mathrm{l} \mathrm{FA/PAMAM} \mathrm{solution} \mathrm{containing} 5 \mu \mathrm{g}$ miR-7 oligonucleotide or liposome dilution. General survival

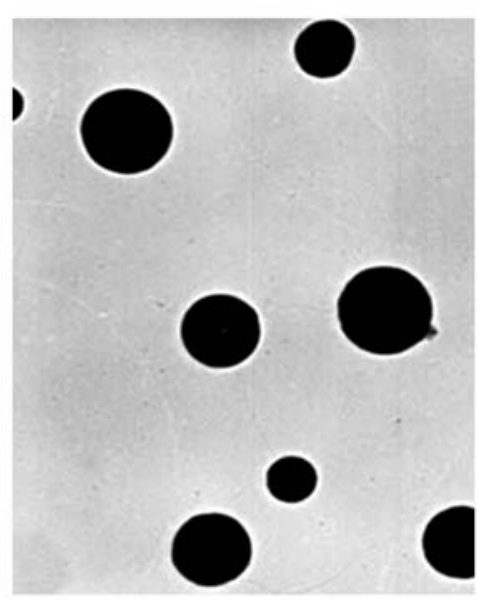

Figure 1. FA/PAMAM morphology using transmission electron microscopy $\left(\mathrm{x} 10^{5}\right)$. FA/PAMAM were solid spheres and the particles were evenly distributed.

of animals was observed and survival duration was recorded. MRI was conducted at 7, 14 and 21 days following cell transplantation and tumor volumes were compared. The animal studies were approved by...

In situ cell death detection. The apoptotic cell death in the tumor specimens of the mice that had died due to the tumor was detected using a cell death detection kit [ApopTag ${ }^{\circledR}$ In Situ Apoptosis Detection kit (S7100), Chemicon International, Inc., Temecula, CA, USA].

Immunohistochemistry. Paraffin-embedded tissue sections were used to examine proliferating cell nuclear antigen, matrix metalloproteinase 2 and matrix metalloproteinase 9 levels. Transfected cells were seeded on coverslips, fixed with $4 \%$ paraformaldehyde (Sigma), treated with $3 \% \mathrm{H}_{2} \mathrm{O}_{2}$ for $10 \mathrm{~min}$ and incubated overnight at $4{ }^{\circ} \mathrm{C}$ with the primary antibodies (1:200 dilutions) overnight at $4^{\circ} \mathrm{C}$, followed by biotin-labeled secondary antibody (1:100 dilutions) for $1 \mathrm{~h}$ at room temperature. Sections were then incubated with avidin-biotin-peroxidase complex and diaminobenzidine, counterstained with hematoxylin and visualized using light microscopy.

Statistical analysis. Data are expressed as the means \pm SD. Data were analyzed using analysis of variance and the $\chi^{2}$ test using SPSS13.0 (Windows). $\mathrm{P}<0.05$ was considered to indicate statistically significant differences.

\section{Results}

FA/PAMAM complex appearance. FA/PAMAM formed solid spheres that were $\sim 50 \mathrm{~nm}$ in diameter and evenly distributed; the PAMAM/miR-7 or PAMAM/nonsense sequence particles were $60-80 \mathrm{~nm}$ in diameter and were evenly distributed (Fig. 1).

Gene transfection efficiency. Using fluorescence microscopy with excitation light of $550 \mathrm{~nm}$, FITC-labeled complex particles appeared green and were distributed in the cell membrane or cytoplasm of U251 cells (Fig. 2). 
Table I. Comparison of U251 glioma cell cycle among groups.

\begin{tabular}{lccrr}
\hline Group & $\begin{array}{c}\mathrm{G}_{1} \text { phase } \\
(\%)\end{array}$ & $\begin{array}{c}\mathrm{S} \text { phase } \\
(\%)\end{array}$ & $\begin{array}{r}\mathrm{G}_{2}-\mathrm{M} \text { phase } \\
(\%)\end{array}$ & $\mathrm{PIx}$ \\
\hline Control & 56.44 & 34.12 & 9.44 & 43.56 \\
Nonsense & 54.27 & 35.49 & 10.24 & 45.73 \\
miR-7 transfection & 72.14 & 14.79 & 13.07 & $27.86^{\mathrm{a}}$ \\
\hline
\end{tabular}

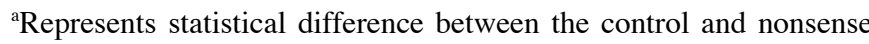
groups $(\mathrm{P}<0.05)$.

miR-7 transfection suppresses U251 glioma cell proliferation. $\mathrm{U} 251$ glioma cells grew rapidly in the control and nonsense sequence groups and there was no significant difference ( $\mathrm{P}>0.05$ ); miR-7 transfection significantly suppressed U251 glioma cell proliferation compared with the control and nonsense sequence groups $(\mathrm{P}<0.05$; Fig. $3 \mathrm{~A})$. The percent of $\mathrm{S}$-phase cells in the control and nonsense sequence groups was significantly more than that in the miR-7 transfection group, $(\mathrm{P}<0.05$; Table I, Fig. 3B). In addition, few apoptotic cells were observed in the control and nonsense sequence groups ( $\mathrm{P}>0.05)$, but the apoptosis rate was significantly increased in the miR-7-transfected cells compared with the control and nonsense sequence groups $(\mathrm{P}<0.05$; Fig. $3 \mathrm{C})$. A larger number of tumor cells in the control $(79.44 \pm 11.34 \%)$ and nonsense sequence groups $(83.67 \pm 9.14 \%)$ migrated to the lower chamber of the migration assay compared with the miR-7-transfected group (31.79 $\pm 6.27 \%)$; $\mathrm{P}<0.05$; Fig. 3D). A large number of cell clones was observed in the control and nonsense sequence groups, with many pseudopodia, but small-sized cell clones

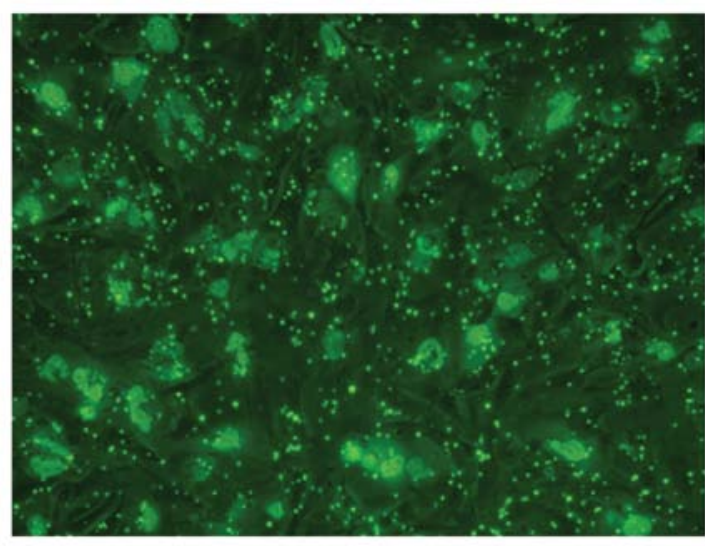

Figure 2. FA/PAMAM-miR-7-transfected U251 glioma cells observed using fluorescence microscopy (magnification, $\mathrm{x} 400$ ). The complex particles were stained green and appeared to be bead or spot-shaped in U251 cell membranes or cytoplasm.

in the absence of pseudopodia were observed in the miR-7 transfection group.

PAMAM increases miR-7 expression and partially suppresses EGFR pathway protein levels. miR-7 expression was low in normal U251 cells and in FA/PAMAM/nonsense-treated cells, with no significant difference $(\mathrm{P}>0.05)$, but it was significantly increased in FA/PAMAM/miR-7-transfected cells $(\mathrm{P}<0.05$; Fig. 4A). In addition, a high level of EGFR and high levels of PI3K and AKT2 were observed in normal U251 cells and in FA/PAMAM/nonsense-treated cells, but EGFR, PI3K and AKT2 levels were significantly decreased in FA/PAMAM/miR-7-transfected cells ( $\mathrm{P}<0.05$; Fig. 4B and $\mathrm{C}$ ).
A

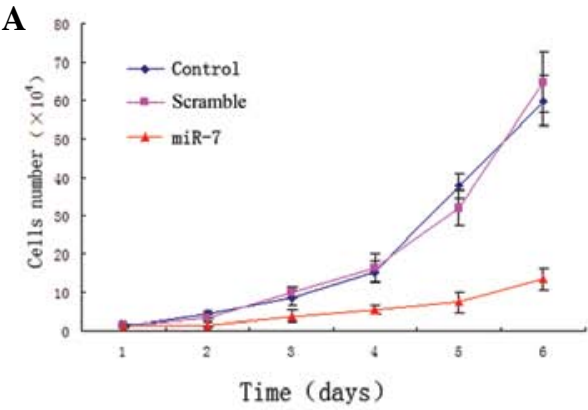

B

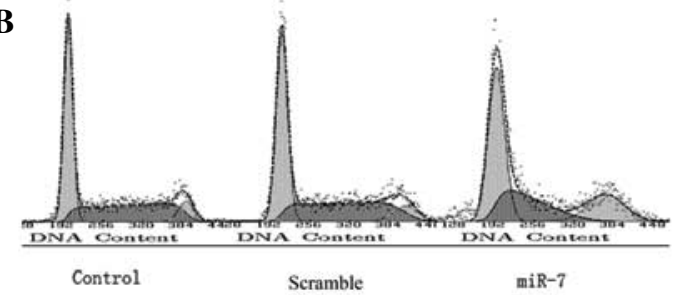

Control

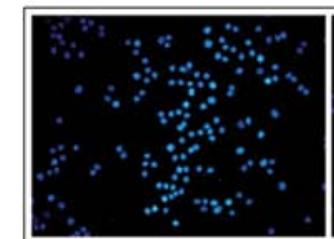

D

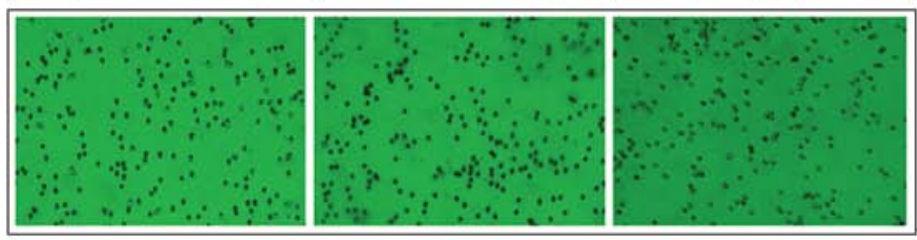

$\mathbf{E}$

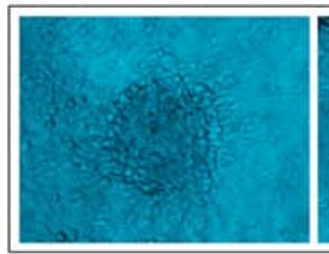

Scramble
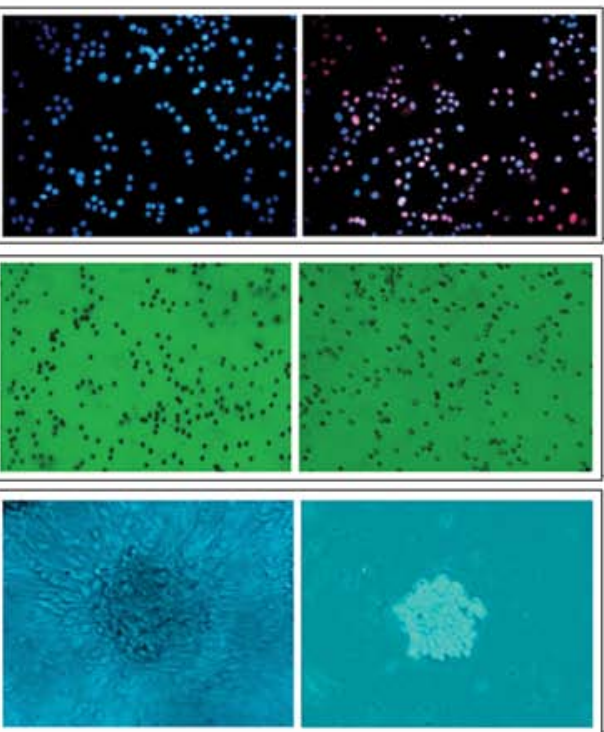

Figure 3. miR-7 transfection suppressed U251 glioma cell proliferation. (A) Cell growth curves show the slowest growth speed of U251 glioma cells was in the miR-7 transfection group. (B) Cell cycle results show that the percent of S-phase cells was significantly less in the miR-7 transfection group compared to the control and nonsense sequence groups. (C) Cell apoptosis results show more apoptotic cells in the miR-7 transfection group compared with the control and nonsense sequence groups. (D) Cell invasion assay revealed decreased invasion ability of U251 glioma cells in the miR-7 transfection group. (E) The ability of clone formation in soft agar was decreased in miR-7-transfected cells. 
A
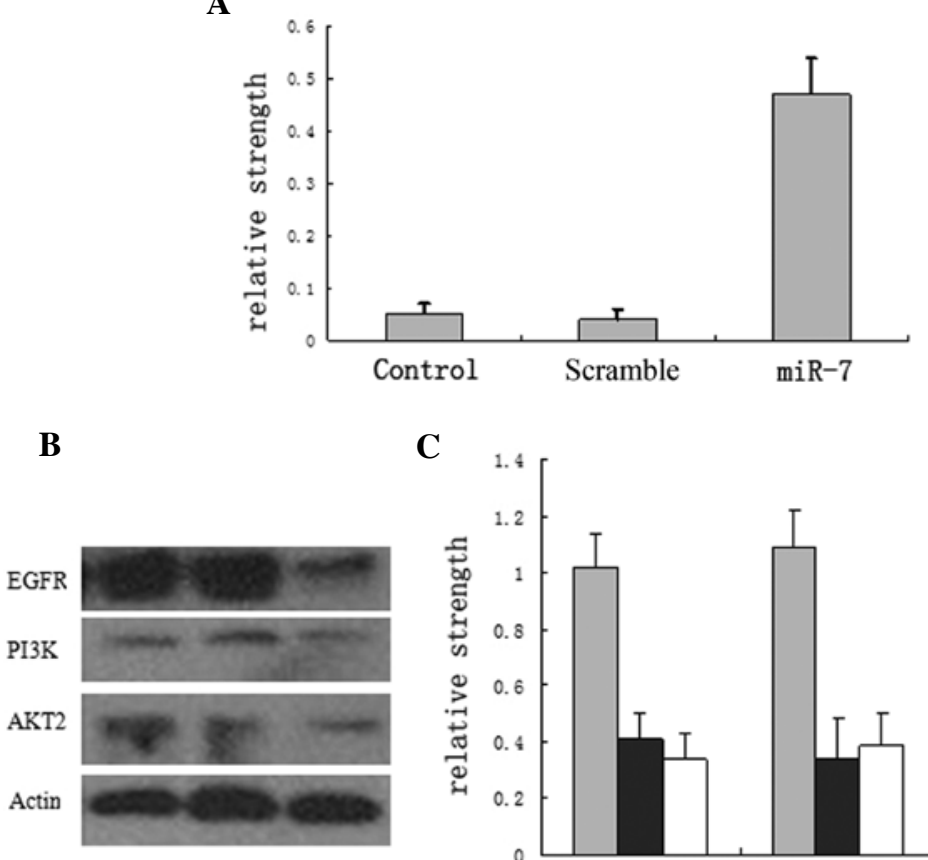

C

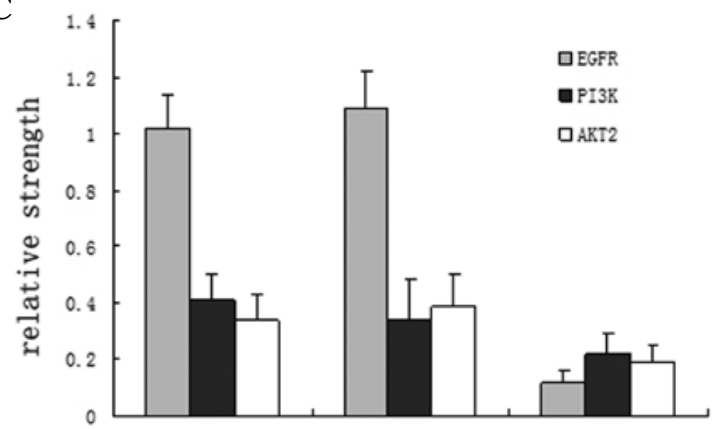

Figure 4. PAMAM increased miR-7 expression and partially suppressed EGFR pathway proteins in U251 glioma cells. (A) RT-PCR showed higher levels of miR-7 expression in FA/PAMAM/miR-7-transfected cells compared with normal U251 cells and FA/PAMAM/nonsense-treated cells. (B) Western blot results showed that PAMAM-mediated miR-7 transfection further suppressed EGFR pathway protein levels. (C) Bar graph of western blot results.
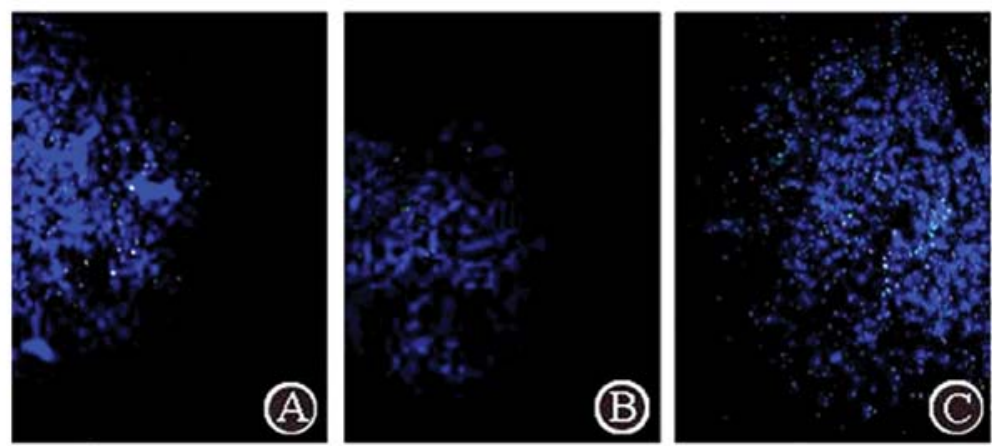

Figure 5. FA/PAMAM/miR-7 complex aggregation in tumors through three administration routes. Blue represents Hoechst 33258-labeled tumor cell nuclei, and green represents FITC-labeled complex (magnification, x100). (A) A few green fluorescence signals were observed in the tumor following caudal vein administration. (B) A few green fluorescence signals were observed in the tumor following carotid artery administration. (C) A large number of green fluorescence signals was observed in the tumor following tumor site administration.

Complex aggregation in the tumor through three administration routes. Using fluorescence microscopy with excitation light of $550 \mathrm{~nm}$, FITC-labeled complex particles exhibited green fluorescence. The FA/PAMAM/miR-7 complex aggregated in the tumor (Fig. 5). Following caudal vein administration, a few positive signals were observed in the tumor, and the positive rate was $15.3 \pm 3.7 \%$ in 10 random high power fields of view, but no positive signals were detected in other regions of the brain. Following carotid artery administration, green fluorescence signals were significantly enhanced in the tumor compared with caudal vein administration $(\mathrm{P}<0.05)$ and the positive rate was $27.4 \pm 5.4 \%$; no positive signals were detected in other regions of the brain. Administration at the tumor site produced the strongest positive signals in the tumor compared with the other two methods and the positive rate was $91.7 \pm 6.1 \%(\mathrm{P}<0.05)$.
PAMAM produces higher levels of miR-7 transfection compared with liposomes. Using fluorescence microscopy with excitation light of $550 \mathrm{~nm}$, FITC-labeled green fluorescence was distributed in the U251 cell membrane or cytoplasm in a bead or spot shape (Fig. 6A). In 10 randomly selected high-power fields of view, liposome transfection efficiency was $51.4 \pm 6.9 \%$ and FA/PAMAM transfection efficiency was $87.6 \pm 7.8 \%$ which was statistically different $(\mathrm{t}=11.6, \mathrm{P}<0.05)$. Only a low level of miR-7 expression was detected in the control group, with a relative expression intensity of $0.09 \pm 0.01$. miR-7 expression was significantly increased in the liposome/ miR-7 group with a relative expression intensity of $0.64 \pm 0.08$; the relative expression intensity was $0.77 \pm 0.06$ in the FA/ PAMAM/miR-7 group. There were significant differences among or between any two groups $(\mathrm{P}<0.05$; Fig. $6 \mathrm{~B})$. 


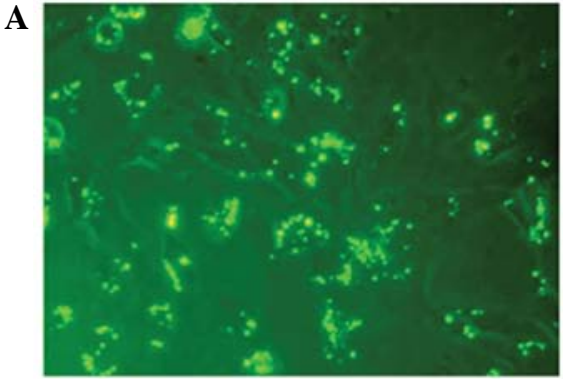

liposome

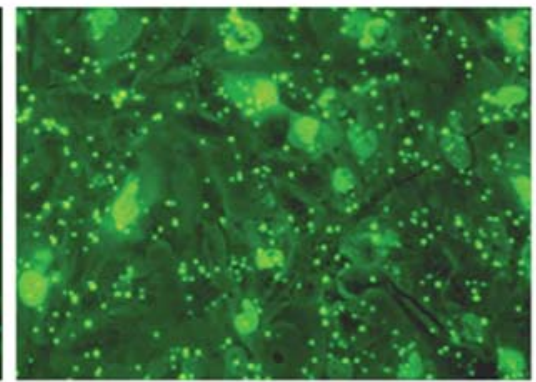

FA/PAMAM

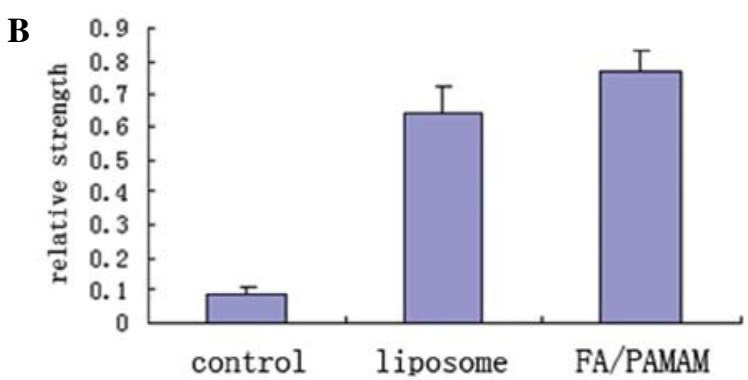

Figure 6. Transfection efficiency was higher for PAMAM than for liposomes. (A) Transfection positive rate in U251 cells using liposomes and PAMAM (magnification, x400). (B) Bar graph.
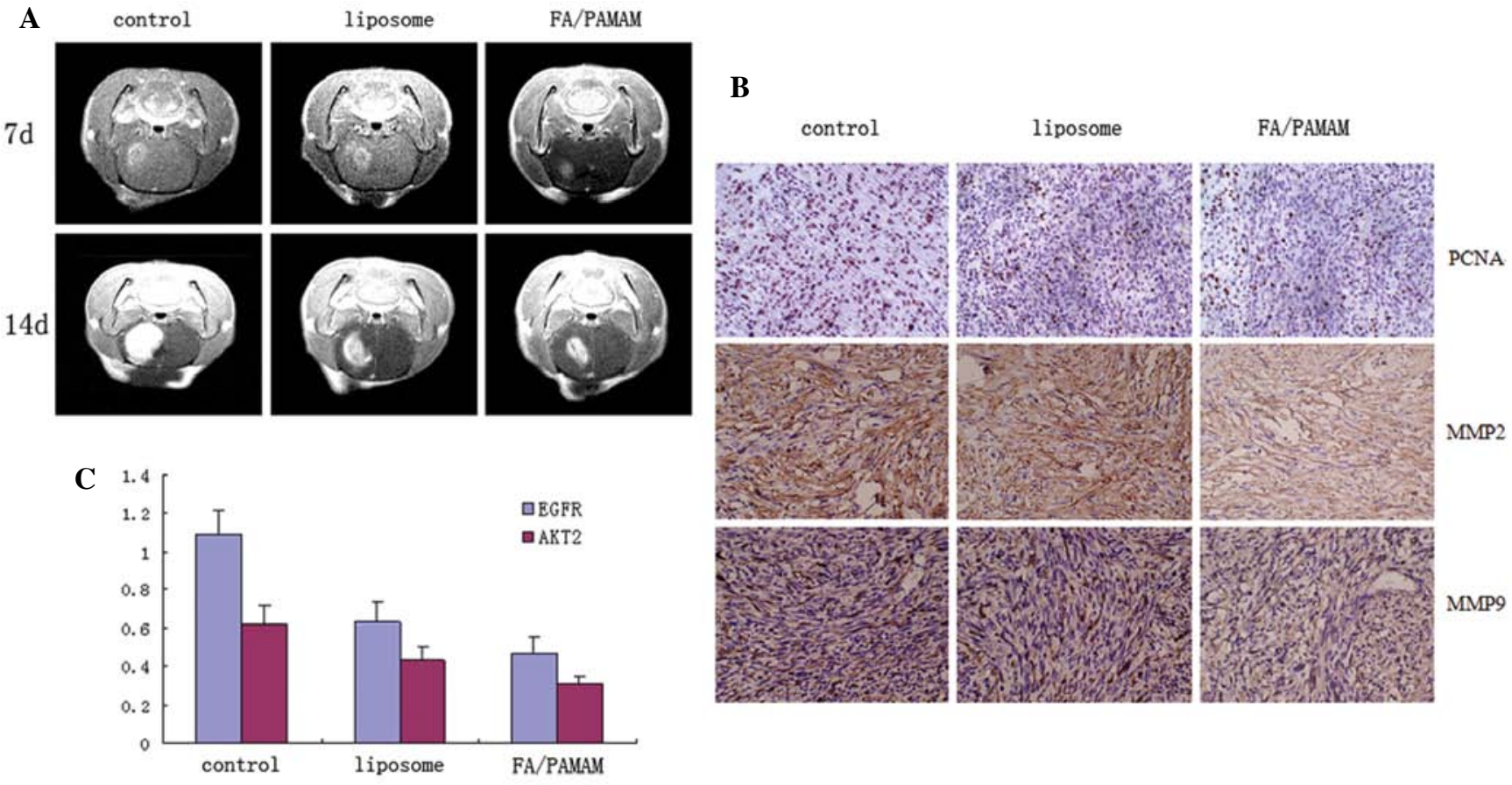

Figure 7. PAMAM/miR-7 suppressed tumor growth more than liposome/miR-7. (A) Tumor growth was significantly suppressed by PAMAM/miR-7 compared to the other groups at 7 and 14 days. (B) Immunohistochemistry showed that PAMAM/miR-7 transfection significantly reduced the levels of glioma cell proliferation and cell invasion marker proteins compared with the control and liposome/miR-7 groups (magnification, x200). (C) Western blot results showed that PAMAM/miR-7 transfection was the most efficient in silenced EGFR pathway genes.

PAMAM/miR-7 suppresses tumor growth more than liposomel $m i R-7$. Mice in the control group exhibited decreased activity, depressed emotion, reduced motion to food and water, and worsened limb hemiplegia. Their mean survival duration was
$16.4 \pm 2.2$ days. The pathological status of mice treated with liposome/miR-7 was delayed and the survival duration was prolonged, with mean survival duration of $19.4 \pm 2.1$ days. The pathological status was significantly delayed in mice treated 
with FA/PAMAM/miR-7 and they survived for $23.5 \pm 2.4$ days on average. There were significant differences among or between any two groups $(\mathrm{P}<0.05)$.

In the control group, intracranial tumor focus was formed at 7 days after cell transplantation, and the volume of the tumor grew to $1 / 2$ the size of the hemisphere at 14 days, with evident edema surrounding the tumor. Intracranial tumor focus was also observed in the liposome/miR-7 group at 7 days after cell transplantation, and the volume of the tumor grew to $1 / 2$ the size of the hemisphere at 14 days, but the volume of the tumor was smaller than that of the control group. Tumor size was smaller in the PAMAM/miR-7 group compared with the control and liposome/miR-7 groups at 7 and 14 days and edema surrounding the tumor improved (Fig. 7A).

The in situ cell apoptosis rate was $5.3 \pm 0.9 \%$ in the control group, $11.4 \pm 2.4 \%$ in the liposome/miR-7 group, and $17.7 \pm 3.7 \%$ in the FA/PAMAM/miR-7 group, with significant differences among or between any two groups $(\mathrm{P}<0.05)$.

Proliferating cell nuclear antigen, matrix metalloproteinase 2 and 9 positive rate was $57.3 \pm 7.4 \%, 45.4 \pm 6.9 \%$ and $55.1 \pm 7.3 \%$, respectively, in the control group, $49.3 \pm 5.9 \%$, $31.7 \pm 7.1 \%$ and $39.4 \pm 6.4 \%$, respectively, in the liposome/miR-7 group, and $34.6 \pm 5.4 \%, 24.5 \pm 4.1 \%$ and $25.4 \pm 5.1 \%$, respectively, in the FA/PAMAM/miR-7 group, with significant differences among or between any two groups $(\mathrm{P}<0.05$; Fig. $7 \mathrm{~B})$.

The EGFR and AKT2 relative expression intensity was $1.09 \pm 0.12$ and $0.62 \pm 0.1$ in the control group, $0.63 \pm 0.11$ and $0.43 \pm 0.07$ in the liposome/miR-7 group, and $0.47 \pm 0.09$ and $0.31 \pm 0.04$ in the FA/PAMAM/miR-7 group, with significant differences among or between any two groups $(\mathrm{P}<0.05$; Fig. 7C).

\section{Discussion}

The PI3K pathway is a classic pathway in glioma EGFR signal transduction: ligand $\rightarrow \mathrm{EGFR} \rightarrow \mathrm{PI} 3 \mathrm{~K} \rightarrow$ protein kinase $\mathrm{C} \rightarrow \mathrm{I} \kappa \mathrm{B}$ kinase, which phosphorylates inhibitory protein $-\kappa \mathrm{B}$, resulting in nuclear factor $\kappa \mathrm{B}$ migration to the nucleus (21). Nuclear factor $\kappa \mathrm{B}$ is an important transcription factor, and the cascade reaction of signal transduction can induce intranuclear DNA synthesis and transformation, impacting glioma cell proliferation, differentiation and migration (22). In the present study, a miR-7 fragment was designed to silence EGFR and was transfected into U251 glioma cells using FA/PAMAM as a vector. The 5' end of the miR-7 fragment was labeled with FITC to mark cell transfection efficiency. Results showed that green fluorescein was evenly distributed in cell membranes or cytoplasm and the positive rate was $87.6 \pm 7.8 \%$. This was significantly higher than liposomemediated miR-7 transfection $(58.5 \pm 5.4 \%)$, indicating the high efficiency of FA/PAMAM. qRT-PCR results suggested that the level of miR-7 was significantly increased $48 \mathrm{~h}$ following FA/PAMAM/miR-7 transfection. Moreover, the levels of three major members of the EGRF pathway, EGFR, PI3K and AKT2, were significantly reduced, indicating that miR-7 transfection can effectively silence the EGFR pathway in glioma cells.

Subsequently, the potential for cell proliferation activity, invasion ability and tumorigenesis was investigated. Results showed that following miR-7 transfection, U251 cell proliferation was significantly decreased, and a high percent of cells was in the $G_{1}$ phase. Moreover, the invasion ability of cells was significantly reduced. Soft agar cell clone formation has frequently been used to simulate semisolid growth environment of the in vivo extracellular matrix and to assess colony growth potential of tumor cells (23). In the present study, the clone formation ability of miR-7 transfected cells was significantly decreased, indicating reduced oncogenicity of miR-7 transfected U251 glioma cells.

In addition, the present study investigated the glioma targeting of FA/PAMAM in vivo. FA/PAMAM/miR-7 administered through the caudal vein, carotid artery or tumor in situ aggregated in the tumor bed in the brain, but not in tissues surrounding the tumor, indicating good targeting of the FA/FA receptor axis. The positive rate of FA/PAMAM/miR-7 aggregation in the tumor bed was $15.3 \pm 3.7 \%, 27.4 \pm 5.4 \%$ and $91.7 \pm 6.1 \%$ with delivery through the caudal vein, carotid artery or tumor in situ, respectively, while the positive rate of liposome/miR-7 aggregation in the tumor bed was $5.4 \pm 0.7 \%, 11.4 \pm 2.1 \%$ and $64.5 \pm 7.4 \%$ through the caudal vein, carotid artery or tumor in situ, respectively, indicating high targeting efficiency of FA/ PAMAM as a small molecule drug carrier. Data comparison also showed significant differences in the amount of drug aggregation in the tumor bed among the three administration routes, the amount of drug was maximal in the tumor in situ group, followed by the carotid artery and then the caudal vein groups. This may result from the drug being retained in some organs or tissues. Therefore, the reduction of drug retainment following vessel perfusion is important in developing small molecule target drugs.

To assess the treatment effect of FA/PAMAM/miR-7 on in vivo and in vitro glioma, the present study utilized liposomes as a control. Results showed that FA/PAMAM significantly improved miR-7 transfection efficiency compared with liposomes, manifested by higher miR-7 expression in transfected $\mathrm{U} 251$ cells. In vivo experiments showed that the survival of animals was prolonged in the FA/PAMAM/miR-7 group compared with the liposome/miR-7 group. In particular, MRI results showed slow glioma proliferation in the early stage, but rapid growth in the middle and later stages in the liposome/miR-7 group, but continuously slow proliferation in the FA/PAMAM/miR-7 group. The second MRI screen (14 days following U251 glioma cell transplantation) showed that the tumor size was significantly smaller in the FA/PAMAM/miR-7 group compared with the control and liposome/miR-7 groups. These results were consistent with the delayed drug release property of FA/PAMAM. Furthermore, to investigate the effect of miR-7 on silencing target genes, the present study detected EGFR and AKT2 protein levels. Results showed better target gene silencing in the FA/PAMAM/miR-7 group, manifested as significantly lower EGFR and AKT2 protein levels compared with the control and liposome/miR-7 groups. The proliferating cell nuclear antigen positive rate can directly reflect tumor cell proliferation activity, matrix metalloproteinase 2 and 9 levels represent tumor cell invasion ability (8). Results from the present study indicate that FA/PAMAM/miR-7 significantly suppressed tumor cell proliferation and invasion ability. As there were no complementary sequences in miR-7 with proliferating cell nuclear antigen, matrix metalloproteinase 2 and 9 , 
we assume that the protein suppression results from EGFR and AKT2 silencing effects.

In conclusion, PAMAM exhibited better gene transfection efficiency and target gene silencing compared with liposomes. Moreover, PAMAM can maintain a longer action to significantly suppress tumor cells. However, FA/PAMAM/miR-7 transfection did not completely remove glioma. The survival duration of animals was only prolonged and the survival rate of 30 days remains very low. Therefore, further investigations are required for glioma gene therapy.

\section{Acknowledgements}

This study was supported by the China National Natural Scientific Fund (81000901), the Tianjin Science and Technology Committee (09JCYBJC09500), the Key Laboratory Project of Tianjin Programs for Science and Technology (10SYSYJC28800), the Key Project of Chinese National Programs for Fundamental Research and Development (973 Program, 2010CB529405), and the Tianjin Health Bureau Science and Technology Projects (2011KZ24).

\section{References}

1. Nakamura M, Shimada K, Nakase H, et al: Clinicopathological diagnosis of gliomas by genotype analysis. Brain Nerve 61: 773-780, 2009 (In Japanese).

2. Mladkova $\mathrm{N}$ and Chakravarti A: Molecular profiling in glioblastoma: prelude to personalized treatment. Curr Oncol Rep 11: 53-61, 2009

3. Huang H, Mahler-Araujo BM, Sankila A, et al: APC mutations in sporadic medulloblastomas. Am J Pathol 156: 433-437, 2000.

4. Gilbertson RJ: Medulloblastoma: signalling a change in treatment. Lancet Oncol 5: 209-218, 2004.

5. Misaki K, Marukawa K, Hayashi Y, et al: Correlation of gammacatenin expression with good prognosis in medulloblastomas. J Neurosurg 102: 197-206, 2005.

6. Ellison DW, Onilude OE, Lindsey JC, et al: beta-Catenin status predicts a favorable outcome in childhood medulloblastoma: the United Kingdom Children's Cancer Study Group Brain Tumour Committee. J Clin Oncol 23: 7951-7957, 2005.

7. Oikonomou E, Barreto DC, Soares B, et al: Beta-catenin mutations in craniopharyngiomas and pituitary adenomas. J Neurooncol 73: 205-209, 2005.
8. Sekine S, Shibata T and Kokubu A: Craniopharyngiomas of adamantinomatous type harbor beta-catenin gene mutations. Am J Pathol 161: 1997-2001, 2002.

9. Gonzalez S, Pisano DG and Serrano M: Mechanistic principles of chromatin remodeling guided by siRNAs and miRNAs. Cell Cycle 7: 2601-2608, 2008.

10. Croce CM: Causes and consequences of microRNA dysregulation in cancer. Nat Rev Genet 10: 704-714, 2009.

11. Novakova J, Slaby O, Vyzula R, et al: MicroRNA involvement in glioblastoma pathogenesis. Biochem Biophys Res Commun 386: $1-5,2009$.

12. Si ML, Zhu S, Wu H, et al: miR-21-mediated tumor growth. Oncogene 26: 2799-2803, 2007.

13. Tong AW and Nemunaitis J: Modulation of miRNA activity in human cancer: a new paradigm for cancer gene therapy? Cancer Gene Ther 15: 341-355, 2008.

14. Kefas B, Godlewski J, Comeau L, et al: microRNA-7 inhibits the epidermal growth factor receptor and the Akt pathway and is down-regulated in glioblastoma. Cancer Res 68: 3566-3572, 2008.

15. Yang H: Nanoparticle-mediated brain-specific drug delivery, imaging, and diagnosis. Pharm Res 27: 1759-1771, 2010.

16. Tomalia DA: A new class of polymers: starburst-denfritic macromolecules. Polym J 17: 117-132, 1985.

17. Malik N, Wiwattanapatapee R, Klopsch R, et al: Dendrimers: relationship between structure and biocompatibility in vitro, and preliminary studies on the biodistribution of 125I-labelled polyamidoamine dendrimers in vivo. J Control Release 65: 133-148, 2000.

18. Huang S, Fu L, Zhang X, et al: Syntheses of polyamidoamine dendrimers starting from a hexadimensional core and application in gene transfer. Science in China Series B: Chemistry 46: 271-279, 2003.

19. Tang MX, Redemann CT and Szoka FC Jr: In vitro gene delivery by degraded polyamidoamine dendrimers. Bioconjug Chem 7 : 703-714, 1996.

20. Luo D, Haverstick K, Belcheva N, et al: Poly(ethylene glycol)-conjugated PAMAM dendrimer for biocompatible, high-efficiency DNA delivery. Macromolecules 35: 3456-3462, 2002.

21. Belda-Iniesta C, de Castro Carpeño J, Sereno M, et al: Epidermal growth factor receptor and glioblastoma multiforme: molecular basis for a new approach. Clin Transl Oncol 10: 73-77, 2008.

22. Loew S, Schmidt U, Unterberg A, et al: The epidermal growth factor receptor as a therapeutic target in glioblastoma multiforme and other malignant neoplasms. Anticancer Agents Med Chem 9: 703-715, 2009.

23. Zi X, Guo Y, Simoneau AR, et al: Expression of Frzb/secreted Frizzled-related protein 3, a secreted Wnt antagonist, in human androgen-independent prostate cancer PC-3 cells suppresses tumor growth and cellular invasiveness. Cancer Res 65: 9762-9770, 2005. 